\title{
D-Cluster Converter Foil for Laser-Accelerated Deuteron Beams: Towards Deuteron-Beam-Driven Fast Ignition
}

\section{Proposal \# DE-SC0006355 \\ (Final Report)}

“Rights in Data - SBIR/STTR Program”

By:

Prime Contractor:

Dr. George H. Miley, PI

NPL Associates, Inc.

912 W. Armory Dr.

Champaign, IL 61821

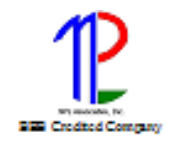

Subcontractor:

Dr. Xiaoling Yang, co-PI

University of Illinois

Dept. of NPRE

216 Talbot Lab,

104 S. Wright Street

Urbana, IL 61801

Date: 10/24/12 


\section{Project Summary}

\section{1) How the research adds to the understanding of the area investigated.}

Fast Ignition (FI) uses Petawatt laser generated particle beam pulse to ignite a small volume called a pre-compressed Inertial Confinement Fusion (ICF) target, and is the favored method to achieve the high energy gain per target burn needed for an attractive ICF power plant. A deuteron beam is especially attractive due to its good focusing capability and the added ("bonus") energy gained as it interacts with the deuterium and tritium in the ICF target during the "hot spot" heating process. However, its use has been curtailed to date by a lack of a suitable deuterium containing converter foil. We proposed to develop a volumetrically-loaded ultra-high-density deuteron deuterium cluster material as the basis for converter-foil for deuteron beam generation. Due to the volumetric loading in the foil, this will provide sufficient energetic deuteron beam flux for "hot spot" ignition, while avoiding the depletion problem encountered by current proton-driven FI foils. In addition, the deuterons will fuse with the ICF D-T while they slow down, providing an extra "bonus" energy gain as well as heating the hot spot. Besides the FI application, this cluster material can also serves as a high yield D-D neutron source, which will help improve homeland security, and medical therapy.

\section{2) The technical effectiveness and economic feasibility of the methods or techniques investigated or demonstrated.}

In the Phase I research, we were able to improve the hydrogen loading ( $\mathrm{H}$ to $\mathrm{Pd}$ ratio) by orders of magnitude, increasing it from $10^{-4}$ to $10^{-2}$ - meeting the original Phase I goal and representing a significant progress towards the beam intensities needed for both FI and pulsed neutron applications. During the previous laser-ion acceleration experiment at LANL, we were able to accelerate deuterons to $\mathrm{MeV}$ energies without removing the surface contamination, although the intensity was reduced by the initial preferential acceleration of protons from the contaminated surface. Thus, with the improved deutrium uptake in our material, we are confident that significantly higher deuteron flux can be accelerated. Once the surface contamination is removed in situ, even higher flux should be achievable. Currently, the techniques that are used to remove surface contamination layer in situ before the laser shot is by an ablative etching or a laser cleaning technique. Both techniques are fairly mature, and can be utilized for our purpose. If deuterium ion driven fast ignition becomes the accepted route to high gain ICF reactors, these converter foils would be an essential component. It would appear that the D-cluster foil will add some cost - order of 10 cents - to the ICF target that is currently to be about 25 cents per target when mass produced. However, the increased energy gains will make up for the added foil-target assembly costs.

\section{3) How the project is otherwise of benefit to the public.}

Another promising near term project for ICF power technology appears to be the ICF-driven fusion-fission hybrid system for burning actinides. NIF staff has begun preliminary studies of this concept through the LIFE project and Ed Moses, Director of NIF, argues that this hybrid design, being less demanding in the ICF technology, will lead to the earliest commercial use of ICF power. A DOE panel was recently convened to prepare a position paper on fusion-fission hybrids and the preliminary report appears quite positive. The cluster type deuterium beam FI approach could potentially play a vital role in this development by allowing a tritium lean target operation which allows a larger fusion burner support ratio and reduces the tritium breeding requirement for these plants. Thus the NPL target manufacturing technology could become a key component in such plants even before the pure electrical ICF plants come on stream. 


\section{Final Scientific/Technical Report}

\section{D-Cluster Converter Foil for Laser-Accelerated Deuteron Beams: Towards Deuteron Beam Driven Fast Ignition}

\section{Project Overview:}

Fast Ignition (FI) uses Petawatt laser generated particle beam pulse to ignite a small volume called a pre-compressed Inertial Confinement Fusion (ICF) target, and is the favored method to achieve the high energy gain per target burn needed for an attractive ICF power plant. Ion beams such as protons, deuterons or heavier carbon ions are especially appealing for FI as they have relative straight trajectory, and easier to focus on the fuel capsule. But current experiments have encountered problems with the "converter-foil" which is irradiated by the Petawatt laser to produce the ion beams. The problems include depletion of the available ions in the convertor foils, and poor energy efficiency (ion beam energy/ input laser energy).

We proposed to develop a volumetrically-loaded ultra-high-density deuteron deuterium cluster material as the basis for converter-foil for deuteron beam generation. The deuterons will fuse with the ICF DT while they slow down, providing an extra "bonus" energy gain in addition to heating the hot spot. Also, due to the volumetric loading, the foil will provide sufficient energetic deuteron beam flux for "hot spot" ignition, while avoiding the depletion problem encountered by current proton-driven FI foils.

After extensive comparative studies, in Phase I, high purity $\mathrm{PdO} / \mathrm{Pd} / \mathrm{PdO}$ foils were selected for the high packing fraction D-Cluster converter foils. An optimized loading process has been developed to increase the cluster packing fraction in this type of foil. As a result, the packing fraction has been increased from $0.1 \%$ to $10 \%$-meeting the original Phase I goal and representing a significant progress towards the beam intensities needed for both FI and pulsed neutron applications. Fast Ignition provides a promising approach to achieve high energy gain target performance needed for commercial Inertial Confinement Fusion (ICF). This is now a realistic goal for near term in view of the anticipated ICF target burn at the National Ignition Facility (NIF) in CA within a year. This will usher in the technology development Phase of ICF after years of research aimed at achieving breakeven experiment. Methods to achieve the high energy gain needed for a competitive power plant will then be a key developmental issue, and our D-cluster target for Fast Ignition (FI) is expected to meet that need.

\section{Provide a comparison of the actual accomplishments with the goals and objectives of the project.}

The primary goal of Phase I was to increase the packing fraction (\#/cc) of clusters in the convertor foil, thus providing higher beam intensities, and test the loaded foil using Trident laser at LANL with surface contamination layer of the foil removed.

After extensive comparative studies, high purity $\mathrm{PdO} / \mathrm{Pd} / \mathrm{PdO}$ foils were selected for the high packing fraction D-Cluster converter foils. An optimized loading process has been developed to increase the cluster packing fraction in this type of foil. Due to some unexpected issues, we were not able to do a

laser test with Trident laser at LANL. However, the packing fraction has been increased from $0.1 \%$ to $10 \%$-meeting the original Phase I goal and representing a significant progress towards the beam intensities needed for both FI and pulsed neutron applications. 


\section{Summarize project activities for the entire period of funding.}

In the Phase I project, we proposed to develop a volumetrically-loaded ultra-high-density deuteron deuterium cluster material as the basis for converter-foil for deuteron beam generation. The volumetric loading should provide sufficient energetic deuteron beam flux for "hot spot" ignition, while avoiding the depletion problem encountered by current proton-driven FI foils. In addition, the deuterons could fuse with the ICF DT while they slow down, providing an extra "bonus" energy gain in addition to heating the hot spot.

Another very important use of the energetic deuteron beam created with cluster-type foils is an intense pulsed neutron source. Due to the close relationship to the cluster foils for FI, a study of this application will be done as part of this project. By accelerating deuterium ions into a deuterated target, $2.45 \mathrm{MeV}$ neutrons formed via the D-D fusion reaction in the target.

The primary goal of Phase I was to increase the packing fraction (\#/cc) of clusters in the convertor foil, thus providing higher deuterium beam intensities for the fast ignition application. We fabricated $\mathrm{PdO} / \mathrm{Pd} / \mathrm{PdO}$ thin foils $(12.5 \mu \mathrm{m})$, and $\mathrm{Pd} / \mathrm{Ni} / \mathrm{Pd}$ multilayer thin films with $\mathrm{Pd}$ as substrate. As $\mathrm{Pd} / \mathrm{Ni} / \mathrm{Pd}$ multilayer thin films have peeling issue when are subjected to electrolysis process, we decided to use the most robust multilayer thin films/foils, $\mathrm{PdO} / \mathrm{Pd} / \mathrm{PdO}$ multilayer foil, as the main material for cluster loading process.

To look for optimized deuterium loading condition and at the same time lower down the cost of the project, we carried out experiments by loading hydrogen into PdO/Pd/PdO thin foils under different experimental conditions, such as different loading cycles and sample purity.

We planned to test the loaded sample with both TPD system and SQUID system to the existence of high density $\mathrm{H}_{2}$ or $\mathrm{D}_{2}$ cluster material. However, because the SQUID system in $\mathrm{U}$ of Illinois has been down, we decided to use a Gas Chromatogram (GC) unit, which serves similar function as TPD system, to double check the TPD testing results.

Figure 1 shows some TPD tests for $12.5 \mu \mathrm{m} \mathrm{PdO} / \mathrm{Pd} / \mathrm{PdO}$ foils with hydrogen loaded under different loading cycles and purities. All the samples were annealed at $300^{\circ} \mathrm{C}$ for one hour to remove loosely bounded hydrogen. From these results, it seems that samples with more loading cycles gives better hydrogen intake than those with less loading cycles. Pd foil with higher purity presents similar curve shape as those with lower purity, and possess similar amount of hydrogen. But the higher amount of hydrogen possesses high binding energy in 99.95\% Pd than in 99.5\% Pd. Thus, for best performance, the use of high purity $\mathrm{Pd}$ is desirable. Figure 2 shows GC test results for Hydrogen loaded PdO/Pd/PdO foils. The resulted $\mathrm{H}: \mathrm{Pd}$ ratio is ca. 0.12 . This sample was not annealed before test, thus it is understandable that this number is an order of magnitude higher than those from TPD test. 


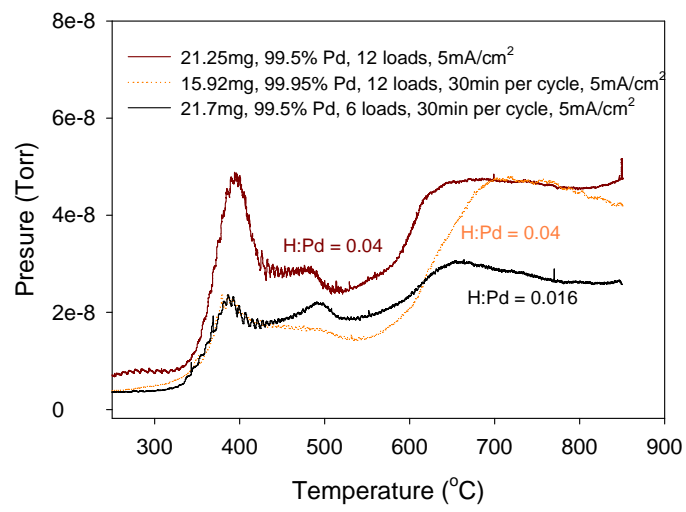

Figure 1 TPD test results for Hydrogen loaded PdO/Pd/PdO foils of different weight.

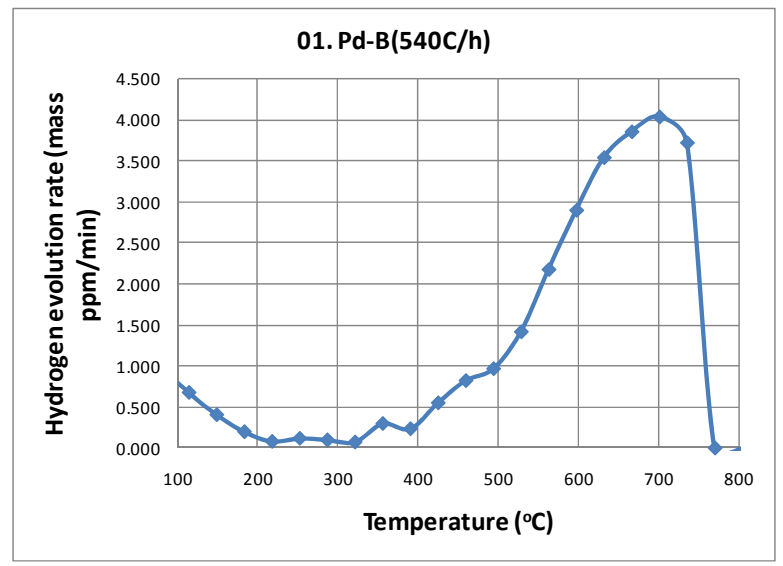

Figure 2 GC test results for Hydrogen loaded $\mathrm{PdO} / \mathrm{Pd} / \mathrm{PdO}$ foils. The $\mathrm{H}: \mathrm{Pd}$ atomic ratio is ca. 0.12. This sample was not annealed before the test.

According to TPD test, the H:Pd ratio under the high temperature peak the 12-loading-cycle cases is ca. 0.02. By comparing with previous results, we found that $\mathrm{H}: \mathrm{Pd}$ ratio of our newly prepared sample has been increased by orders of magnitude - from $10^{-4}$ to $10^{-2}$. (Lipson et al. "Transport and magnetic anomalies below $70 \mathrm{~K}$ in a hydrogen-cycled Pd foil with a thermally grown oxide”, Phys. Rev. B 72, 212507 (2005)). This is a big step forward.

In the later experiments, we studied the effect of current density during electrolysis loading step on $\mathrm{H}$ to Pd ratio. In addition to the current density, $5 \mathrm{~mA} / \mathrm{cm}^{2}$, that we have been using, we have investigated two more hydrogen loading current densities: $1.5 \mathrm{~mA} / \mathrm{cm}^{2}, 2.5 \mathrm{~mA} / \mathrm{cm}^{2}$, to see if lower currents (i.e. slower loading) can lead to more uniform cluster distribution, and thus more hydrogen uptake. We found that higher current density gives a slightly higher hydrogen uptake, but the difference from that with lower current density is not that significant. However, the loading time with a higher current density is much less than that required with a lower current density, making this process more time efficient. Thus, we have elected to stay with the $5 \mathrm{~mA} / \mathrm{cm}^{2}$ in further studies. 
We also studied the effect of the number of loading cycles on the hydrogen uptake of the high purity Pd foils. The results are shown in Figure 3. It can be seen that the sample with 6 loading cycles has hydrogen uptake significantly higher than that with either 12 or 3 loading cycles, indicating there is possibly an optimum number of loading cycles for the high purity samples. This is different from the low purity ones.

In conclusion, in Phase I research, high purity $\mathrm{PdO} / \mathrm{Pd} / \mathrm{PdO}$ foils were selected for the high packing fraction D-Cluster converter foils. An optimized loading

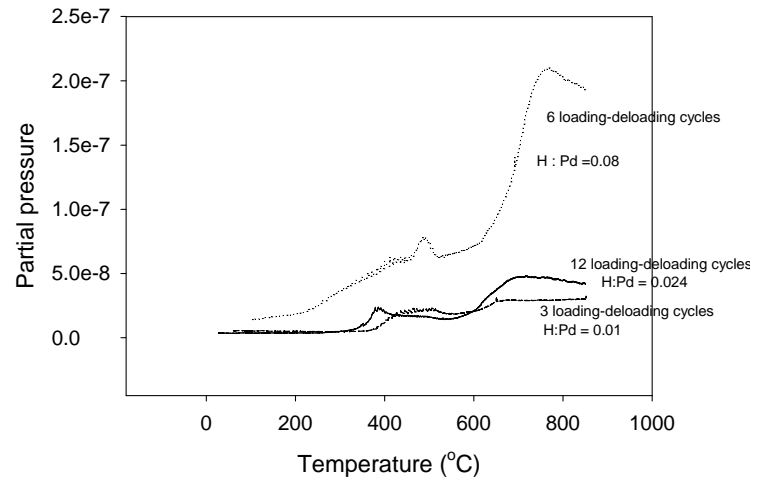

Figure 3 TPD test results for Hydrogen loaded $\mathrm{PdO} / \mathrm{Pd} / \mathrm{PdO}$ foils. The Pd purity is $99.95 \%$. process has been developed to increase the cluster packing fraction in this type of foil. As a result, the packing fraction has been increased from $0.1 \%$ to $10 \%$-meeting the original Phase I goal and representing a significant progress towards the beam intensities needed for both FI and pulsed neutron applications. Due to some unexpected scheduling issues, we were only able to perform a preliminary laser test with the TRIDENT laser at LANL. However, the main objective of achieving improved convertor foil cluster densities has been achieved and this deviation from the project schedule did not affect the project result.

\section{Identify products developed under the award and technology transfer activities, such as:}

a. Publications (list journal name, volume, issue), conference papers, or other public releases of results. If not provided previously, attach or send copies of any public releases to the DOE Program Manager identified in Block 15 of the Assistance Agreement Cover Page;

X.Yang, G. H. Miley, K. A. Flippo, H. Hora (2012), Hot spot heating process estimate using a laser-accelerated quasi-maxwellian deuteron beam, Laser and particle beams, 30(1), 31-38

X.Yang, G. H. Miley, K. A. Flippo, H. Hora (2011), Energy enhancement for deuteron beam fast ignition of a precompressed inertial confinement fusion target, Physics of Plasmas 18(3), 032703

X. Yang et al., (2011), D-Cluster Converter Foil for Laser-Accelerated Deuteron Beams: Towards Deuteron-Beam-Driven Fast Ignition, Fusion Science and Technology, 60 (2), 615-619

b. Web site or other Internet sites that reflect the results of this project; N/A

c. Networks or collaborations fostered; N/A 
d. Technologies/Techniques;

Produce D-cluster converter foil with increased packing fraction by electrochemical loading Deuterium with controlled current density.

e. Inventions/Patent Applications, licensing agreements;

Patent Application in Process - Title "Intense Pulsed Neutron Source using Cluster Foil" Inventors : Dr. George H. Miley, Dr. Xiaoling Yang.

f. Other products, such as data or databases, physical collections, audio or video, software or netware, models, educational aid or curricula, instruments or equipment.

N/A

7. For projects involving computer modeling, provide the following information with the final report: - N/A 